\title{
A serum amyloid A-positive hepatocellular neoplasm arising in alcoholic cirrhosis: a previously unrecognized type of inflammatory hepatocellular tumor
}

\author{
Motoko Sasaki ${ }^{1}$, Norihide Yoneda ${ }^{1,2}$, Seiko Kitamura ${ }^{3}$, Yasunori Sato ${ }^{1}$ and \\ Yasuni Nakanuma ${ }^{1}$ \\ ${ }^{1}$ Department of Human Pathology, Kanazawa University Hospital, Kanazawa, Japan; ${ }^{2}$ Department of \\ Radiology, Kanazawa University Graduate School of Medicine, Kanazawa, Japan and ${ }^{3}$ Diagnostic Pathology \\ Section, Kanazawa University Hospital, Kanazawa, Japan
}

\begin{abstract}
Hepatocellular adenoma usually arises in the absence of significant fibrosis. Herein, we report seven patients with serum amyloid A-positive hepatocellular neoplasm, which shares features with inflammatory hepatocellular adenoma arising in alcoholic cirrhosis. Seven patients (two women and five men, age range 41-67 years) with hypervascular hepatocellular nodules associated with alcoholic cirrhosis were retrieved from our pathological files (1997-2011). The hepatocellular nodules were multiple $(>3)$ in all patients and 17 nodules were histologically examined. We surveyed the immunoreactivity for serum amyloid A, glutamine synthetase, and glypican-3 in the hepatocellular nodules and control lesions, including 5 focal nodular hyperplasia, 18 dysplastic nodules, and 54 hepatocellular carcinomas in various background diseases. In all, 15 of 17 nodules showed strong and distinct immunoreactivity for serum amyloid A, sharing features with inflammatory hepatocellular adenoma. The serum amyloid A-positive hepatocellular neoplasms showed increased cellular density, inflammatory infiltrate, sinusoidal dilatation, and ductular reaction to various degrees. Although about a half of dysplastic nodules and hepatocellular carcinomas showed focal immunoreactivity for serum amyloid $A$, the extent of serum amyloid A expression was significantly higher in serum amyloid A-positive hepatocellular neoplasms, than in control nodules. The serum amyloid A-positive hepatocellular neoplasms did not show the overexpression of glutamine synthetase or immunoreactivity for glypican-3. In contrast, most hepatocellular carcinomas showed the overexpression of glutamine synthetase and immunoreactivity for glypican-3, irrespective of background diseases. In conclusion, this study highlights a characteristic group of hepatocellular neoplasms arising in alcoholic cirrhosis, which share features with inflammatory hepatocellular adenomas. These serum amyloid Apositive hepatocellular neoplasms may be a new type of inflammatory hepatocellular tumors in alcoholic patients. Modern Pathology (2012) 25, 1584-1593; doi:10.1038/modpathol.2012.114; published online 6 July 2012
\end{abstract}

Keywords: alcoholic cirrhosis; dysplatic nodule; hepatocellular carcinoma; inflammatory hepatocellular adenoma; serum amyloid A protein

Hepatocellular adenomas are benign monoclonal tumors occurring essentially in young women taking oral contraceptives. ${ }^{1}$ In all, $85 \%$ of cases occur in young women; hepatocellular adenoma is rare in

Correspondence: Professor Y Nakanuma, MD, Department of Human Pathology, Kanazawa University Graduate School of Medicine, Kanazawa 920-8640, Japan.

E-mail: pbcpsc@kenroku.kanazawa-u.ac.jp

Received 13 March 2012; revised 14 May 2012; accepted 14 May 2012; published online 6 July 2012 children, men, and the elderly. ${ }^{1-5}$ Hepatocellular adenoma represents a heterogeneous entity, recently subclassified into several groups according to genotype and phenotype.$^{2-6}$ Genotyping has allowed the identification of three subtypes: hepatocyte nuclear factor $1 \alpha$-inactivated (35-50\% of cases), $\beta$ catenin-activated (15-18\% of cases), and inflammatory hepatocellular adenomas (40-55\% of cases), which could be identified by immunohistochemistry on paraffin-embedded material. ${ }^{2-6}$ The incidence of hepatocellular adenoma is about 3-4 per 100000 
people in Europe and North America ${ }^{1}$ but is lower in Asian countries, including Japan. The lower incidence of hepatocellular adenoma may be related to the lower prevalence of oral contraceptives in Asian countries, including Japan. ${ }^{7}$

In our recent study on the subtypes and clinicopathological features of hepatocellular adenomas in Japan, we disclosed that inflammatory hepatocellular adenomas characterized by distinct immunoreactivity for serum amyloid A arising in men with alcoholic liver disease may be a major subtype of hepatocellular adenomas in Japan. ${ }^{8}$ The study disclosed different clinicopathological features of hepatocellular adenomas in Japan from those in Europe and North America. ${ }^{8}$ In the study, we examined 14 hepatocellular adenomas (7 women) and identified 1 hepatocyte nuclear factor $1 \alpha$-inactivated hepatocellular adenoma (7\%), $2 \beta$-catenin-activated hepatocellular adenomas (14\%), 7 inflammatory hepatocellular adenomas (50\%), and 4 unclassified hepatocellular adenomas $(29 \%){ }^{8}$ It should be noted that half of all hepatocellular adenomas occur in men and hepatocyte nuclear factor $1 \alpha$-inactivated hepatocellular adenoma showed rather a low incidence. Interestingly, four inflammatory hepatocellular adenoma patients (one woman) were heavy alcohol drinkers and one had alcoholic steatofibrosis and three had alcoholic cirrhosis in our previous study. ${ }^{8}$

Hepatocellular adenomas usually arise in the absence of significant fibrosis; therefore, a liver lesion with a solid mass arising in a fibrotic/cirrhotic background is not thought to be a hepatocellular adenoma according to the diagnostic algorithm proposed in the WHO classification 2010. ${ }^{2}$ There has been no other study describing hepatocellular adenomas arising in advanced liver diseases including alcoholic cirrhosis, to our knowledge. Our previous study ${ }^{8}$ highlighted a group of hepatocellular adenomas, which are in conflict with the concept of 'hepatocellular adenomas'; therefore, more detailed analysis is mandatory to establish this unusual type of hepatocellular adenoma. Focusing on this issue, we have further surveyed and analyzed hepatocellular nodular lesions arising in alcoholic cirrhosis. Herein, we report seven patients with serum amyloid A-positive hepatocellular neoplasm with features of inflammatory hepatocellular adenoma arising in alcoholic cirrhosis. Furthermore, we examined the immunoreactivity for serum amyloid A, glutamine synthetase, and glypican-3 in control groups including focal nodular hyperplasia, dysplastic nodules, and hepatocellular carcinoma in patients with various background liver diseases.

\section{Patients and methods}

\section{Patients}

Seven patients (two women and five men, age ranged 41-67 years; mean \pm s.d., $52.9 \pm 9.7$ years) with hepatocellular nodules associated with alcoholic cirrhosis were retrieved from our pathological files (1997-2011) (Table 1). All patients were heavy drinkers and had been diagnosed with alcoholic cirrhosis. Hypervascular hepatocellular nodules were detected during follow-up. There was no rapid wash-out precluding a diagnosis of hepatocellular carcinoma. Hepatocellular nodules were multiple $(>3)$ in all patients and their size ranged $4-35 \mathrm{~mm}$ (mean \pm s.d.: $12 \pm 8 \mathrm{~mm}$ ) (Table 1). Liver needle biopsy was performed for six patients for diagnosis and one patient underwent liver transplantation. Seventeen nodules were histologically examined. The original diagnosis of these nodules was hyperplastic hepatocellular nodule for seven, dysplastic nodule (adenomatous hyperplasia) for eight and inflammatory hepatocellular adenoma for two.

For comparison, we also examined 5 focal nodular hyperplasias, 18 dysplastic nodules, and 54 hepatocellular carcinomas in patients with alcoholic liver disease $(n=18)$, non-alcoholic fatty liver disease $(n=7)$, and viral hepatitis $(n=29)$ (Table 2). All of these specimens were obtained from our pathological files (1997-2011) and their clinicopathological back-

Table 1 Summary of the patients with serum amyloid A-positive neoplasms and alcoholic cirrhosis

\begin{tabular}{|c|c|c|c|c|c|c|c|}
\hline & Patient 1 & Patient 2 & Patient 3 & Patient 4 & Patient 5 & Patient 6 & Patient 7 \\
\hline Age/sex & $56 / \mathrm{M}$ & $50 / \mathrm{M}$ & $41 / \mathrm{F}$ & $53 / \mathrm{M}$ & $67 / \mathrm{M}$ & $61 / \mathrm{M}$ & $41 / \mathrm{F}$ \\
\hline Oral contraceptives & - & - & Not available & - & - & - & Not available \\
\hline Alcohol > $>40 \mathrm{~g} /$ day & + & + & + & + & + & + & + \\
\hline Vascularity (image study) & Hyper & Hyper & Hyper & Hyper & Hyper & Hyper & Hyper \\
\hline Background liver & Al-cirrhosis & Al-cirrhosis & Al-cirrhosis & Al-cirrhosis & Al-cirrhosis & Al-cirrhosis & Al-cirrhosis \\
\hline Steatosis & - & Mild & - & - & Mild & - & - \\
\hline \multicolumn{8}{|l|}{ Tumor } \\
\hline Tumor size (mean \pm s.d.) & $15 \mathrm{~mm}$ & $\begin{array}{c}6-35 \mathrm{~mm} \\
(13 \pm 11)\end{array}$ & $\begin{array}{c}4-12 \mathrm{~mm} \\
(8 \pm 4)\end{array}$ & $10 \mathrm{~mm}$ & $\begin{array}{c}19-23 \mathrm{~mm} \\
(21 \pm 2)\end{array}$ & $\begin{array}{c}2-10 \mathrm{~mm} \\
(7 \pm 5)\end{array}$ & $10 \mathrm{~mm}$ \\
\hline No. of tumors & $>3$ & $>6$ & $>3$ & $>3$ & $>3$ & $>3$ & $>3$ \\
\hline No. examined & 1 & 6 & 3 & 1 & 2 & 1 & 2 \\
\hline No. of SAA-positive nodules & 1 & 6 & 1 & 1 & 2 & 1 & 2 \\
\hline Association of HCC & - & - & - & - & - & - & - \\
\hline
\end{tabular}

Abbreviations: Al-cirrhosis, alcoholic cirrhosis; F, female; HCC, hepatocellular carcinoma; M, male; SAA, serum amyloid A. 
Table 2 Patients with serum amyloid A-positive neoplasms in alcoholic cirrhosis and control benign and malignant hepatic nodules

\begin{tabular}{|c|c|c|c|c|c|c|c|c|c|c|c|}
\hline \multirow[t]{2}{*}{ Diseases } & \multirow[t]{2}{*}{$\mathrm{n}$} & \multicolumn{2}{|c|}{ Age } & \multicolumn{2}{|c|}{ Gender } & \multicolumn{3}{|c|}{$\begin{array}{c}\text { Serology for } \\
\text { hepatitis viruses }\end{array}$} & \multicolumn{3}{|c|}{$\begin{array}{l}\text { Background } \\
\quad \text { liver }\end{array}$} \\
\hline & & Range & Mean \pm s.d. & $M$ & $F$ & $B$ & $C$ & $B C-$ & FO & $F 1,2$ & $F 3,4$ \\
\hline $\begin{array}{l}\text { SAA-positive neoplasm in } \\
\text { alcoholic cirrhosis }\end{array}$ & 7 & $41-67$ & $52.7 \pm 9.7$ & 5 & 2 & 0 & 0 & 7 & 0 & 0 & 7 \\
\hline Focal nodular hyperplasia & 5 & $18-65$ & $37.8 \pm 16.6$ & 2 & 3 & 0 & 0 & 5 & 4 & 1 & 0 \\
\hline Dysplastic nodule & 18 & $52-83$ & $58.5 \pm 9.7$ & 14 & 4 & 6 & 6 & 6 & 0 & 0 & 18 \\
\hline HCC, alcoholic & 18 & $60-86$ & $70.6 \pm 7.1$ & 17 & 1 & 0 & 0 & 18 & 0 & 2 & 16 \\
\hline HCC, NAFLD & 7 & $68-83$ & $78.0 \pm 6.4$ & 6 & 1 & 0 & 0 & 7 & 0 & 0 & 7 \\
\hline HCC, viral & 29 & $44-76$ & $64.8 \pm 9.3$ & 29 & 0 & 8 & 21 & 0 & 0 & 4 & 25 \\
\hline
\end{tabular}

Abbreviations: F, female; F0-4, degree of fibrosis (F0, none; F1, 2, mild-to-moderate; F3, 4, pre-cirrhosis and cirrhosis); HCC, hepatocellular carcinoma; M, male; NAFLD, non-alcoholic fatty liver disease; SAA, serum amyloid A.

Table 3 Summary of histologic and immunohistochemical features of the patients with serum amyloid A-positive neoplasms and alcoholic cirrhosis

\begin{tabular}{|c|c|c|c|c|c|c|c|}
\hline & Patient 1 & Patient 2 & Patient 3 & Patient 4 & Patient 5 & Patient 6 & Patient 7 \\
\hline \multicolumn{8}{|l|}{ Histologic feature } \\
\hline Steatosis & - & + & - & - & - & - & - \\
\hline Sinusoidal dilatation & +++ & ++ & + & ++ & + & + & ++ \\
\hline Ductular reaction & + & ++ & + & ++ & + & + & + \\
\hline Bile duct loss & ++ & ++ & ++ & ++ & ++ & ++ & ++ \\
\hline Abnormal thick arteries & - & - & - & - & - & - & - \\
\hline Inflammatory reaction & ++ & ++ & $-1+$ & ++ & + & + & ++ \\
\hline Cytological abnormalities & - & - & - & - & $--1+$ & - & - \\
\hline Remodeling & + & $-/+$ & - & - & - & - & - \\
\hline \multicolumn{8}{|l|}{ Immunohistochemistry } \\
\hline LFABP & + & + & + & + & + & + & + \\
\hline Glutamine synthetase & Focal $^{\text {a }}$ & Focal $^{\text {a }}$ & Focal $^{\text {a }}$ & Focal $^{\text {a }}$ & Focal $^{\text {a }}$ & Focal $^{\text {a }}$ & Focal $^{\mathrm{a}}$ \\
\hline$\beta$-Catenin (nuclear) & - & - & - & - & - & - & - \\
\hline Serum amyloid A & + & + & + & + & + & + & + \\
\hline Glypican-3 & - & - & - & - & $-{ }^{\mathrm{b}}$ & - & - \\
\hline
\end{tabular}

Abbreviations: LFABP, liver fatty acid-binding protein; Grading: +, mild; ++, moderate; +++, severe; -, absent;

${ }^{\mathrm{a}}$ Several GS-positive cells around veins.

${ }^{\mathrm{b}}$ A few glypican-3-positive cells.

grounds are shown in Table 2. Histological diagnosis of hepatocellular carcinoma was based on increased cellular density, cellular atypia, and reduction of reticulum fibers. Histological diagnosis of dysplatic nodules was based on increased cellular density, mild cellular atypia, no reduction of reticulum fibers, and lack of features of inflammatory hepatocellular adenoma, such as a ductular reaction.

Liver tissue samples were fixed in 10\% neutralbuffered formalin and embedded in paraffin. More than 20 serial sections, $4 \mu \mathrm{m}$ thick, were cut from each block. Several were processed routinely for histopathologic study, and the remainder was processed for the following immunohistochemistry.

\section{Immunohistochemistry}

We used immunohistochemical markers such as liver fatty acid-binding protein, glutamine synthetase, $\beta$-catenin, and serum amyloid $A$ for the classification of hepatocellular adenomas. We also used glypican-3 as a marker of hepatocellular carcinoma. ${ }^{9}$ Immunohistochemistry was performed as described previously, ${ }^{10}$ using the primary antibodies; liver fatty acid-binding protein (rabbit polyclonal; Abcam, Cambridge, UK, 1:50 dilution), glutamine synthetase (mouse monoclonal, clone GS6; Millipore, Billerica, MA, USA, 1:200 dilution), $\beta$-catenin (mouse monoclonal, clone 14; BD Transductions, San Jose, CA, USA; 1:200 dilution), serum amyloid A (mouse monoclonal; clone mc1, Dako, Glustrup, Denmark, 1:100 dilution), and glypican-3 (mouse monoclonal, clone 1G12; Nichirei, Tokyo, Japan, pre-diluted). In brief, after pre-treatment using a microwave with citrate buffer $(\mathrm{pH} 6), 95^{\circ} \mathrm{C}$, for $20 \mathrm{~min}$, blocking endogenous peroxidase, sections were incubated with the primary antibody at $4{ }^{\circ} \mathrm{C}$ overnight. The Envision + solution for mouse and rabbit (Dako) was then applied for $30 \mathrm{~min}$ at room temperature. The reaction products were visualized using 3-3'-diaminobenizidine tetrahydrochloride 
(Sigma Chemical, St Louis, MO, USA) and $\mathrm{H}_{2} \mathrm{O}_{2}$. The sections were then lightly counterstained with hematoxylin. Similar dilution of the control mouse or rabbit Immunoglobulin G (Dako) was applied instead of the primary antibody as a negative control. Positive and negative controls were routinely included.

The extent of immunoreactivity for serum amyloid A and glutamine synthetase was semiquantitatively assessed as follows: 0 , positive cells; $1,<30 \% ; 2$, $30-70 \% ; 3,>70 \%$. When the immunoreactivity of glutamine synthetase was assessed as 2 or 3 , it was regarded as glutamine synthetase overexpression.

\section{Statistical Analysis}

Numerical data are presented as the mean \pm s.d. Data from different groups were compared using one-way analysis of variance and examined with the Kruskal-Wallis test followed by Dunn's posttest. The results were considered significant if the $P$-value was $<0.05$.

\section{Results}

\section{Summary of the Patients with Serum Amyloid A- Positive Hepatocellular Neoplasms}

Tables 1 and 3 summarize the clinicopathological features and the histological and immunohistochemical findings in seven patients with serum amyloid A-positive hepatocellular neoplasms, respectively. Figure 1 shows the representative histology and immunohistochemical findings in serum amyloid A-positive hepatocellular neoplasms.
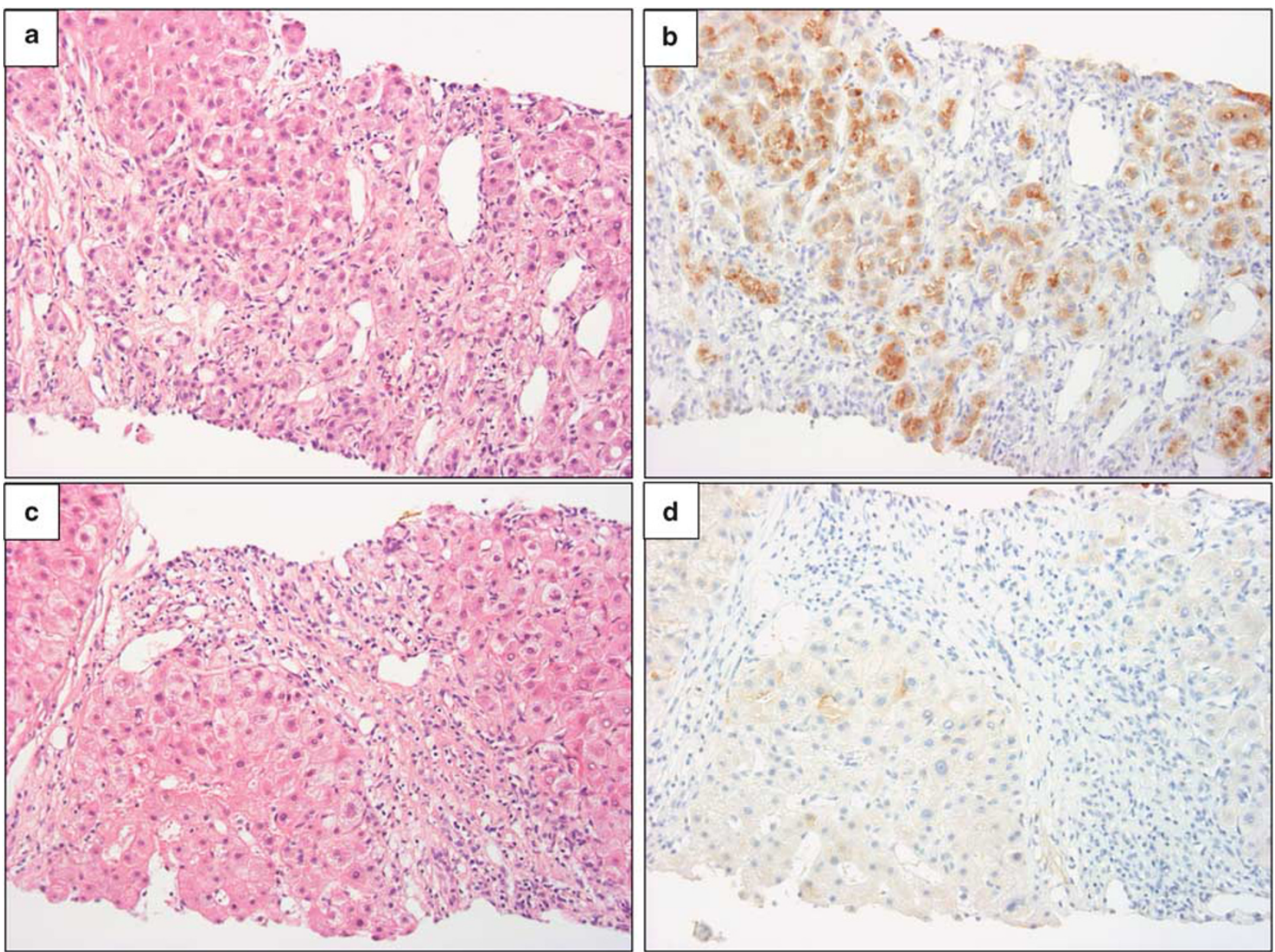

Figure 1 Serum amyloid A-positive hepatocellular neoplasms sharing features with inflammatory hepatocellular adenoma arising in alcoholic cirrhosis. (a) Serum amyloid A-positive nodule in patient 4. Inflammatory cells infiltration, focal sinusoidal dilatation, and ductular reaction are seen in the tumor. HE, $\times 100$. (b) Serum amyloid A-positive nodule in patient 4 (same region as a). The cells in the nodule show extensive granular immunoreactivity for serum amyloid A. Immunostaining for serum amyloid A, $\times 200$. (c) Background liver in patient 4 shows established micronodular cirrhosis consistent with alcoholic cirrhosis. (d) Background liver in patient 4 (same region as c). The background liver shows negative immunoreactivity for serum amyloid A. Immunostaining for serum amyloid A, $\times 200$. (e) Serum amyloid A-positive nodule in patient 1. Marked sinusoidal dilatation, ductular reaction and inflammatory cell infiltration ( ${ }^{*}$ ) are seen in the tumor. HE, $\times 200$. (f) Serum amyloid A-positive nodule in patient 1 . The cells in the serum amyloid A-positive nodule show extensive immunoreactivity for serum amyloid A. Immunostaining for serum amyloid A, $\times 200$. (g) The cells in the serum amyloid A-positive nodule show increased cellular density and few cellular atypia. HE, $\times 400$, patient 4 . 

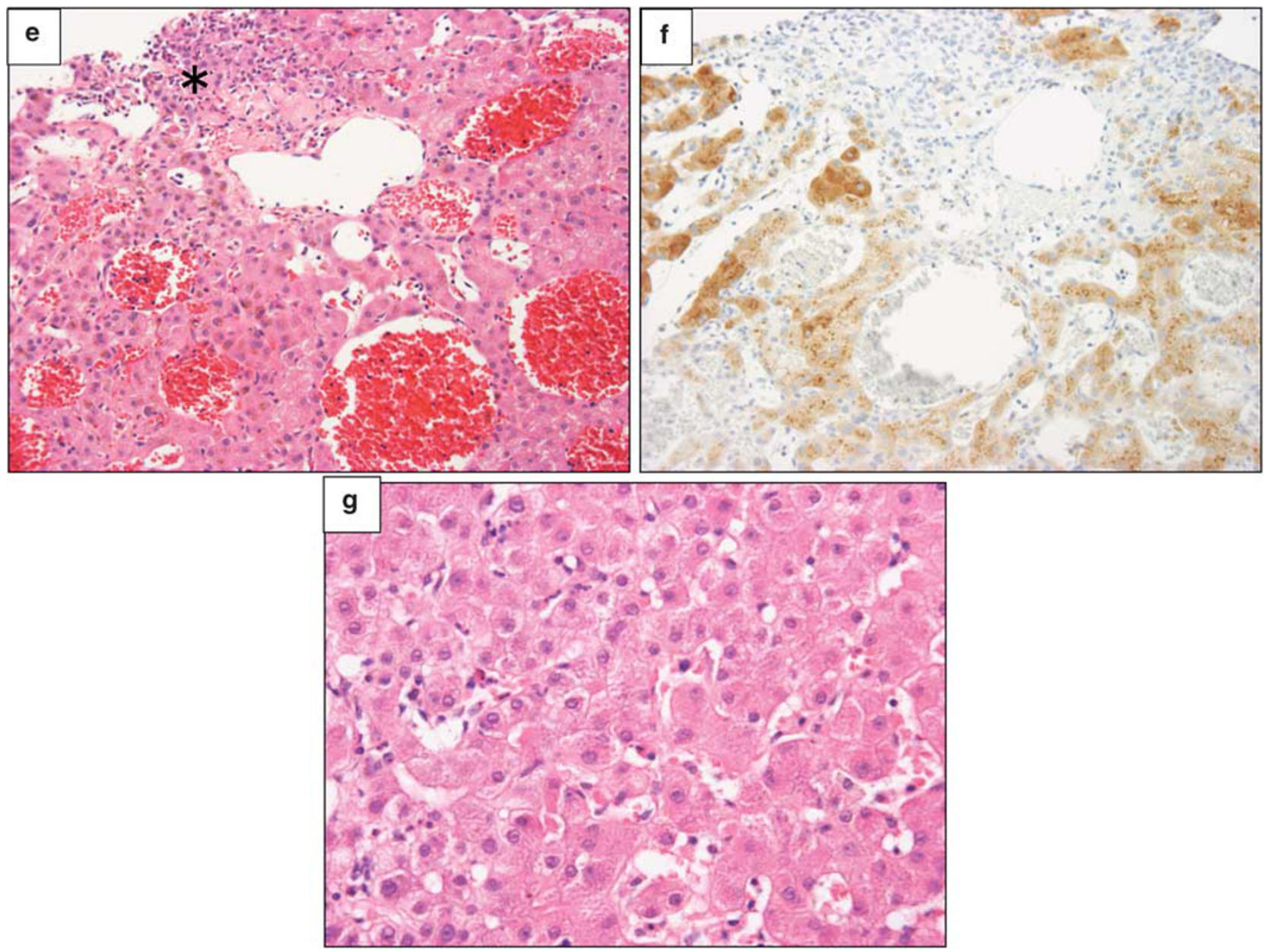

Figure 1 Continued.

Table 4 The expression of serum amyloid A, glutamine synthetase, and glypican-3 in serum amyloid A-positive neoplasms in alcoholic cirrhosis and control hepatic nodules

\begin{tabular}{|c|c|c|c|c|c|c|}
\hline & $\mathrm{n}$ & $\begin{array}{l}\text { SAA, nodule } \\
3 / 2 / 1 / 0\end{array}$ & $\begin{array}{c}\text { SAA, } \\
\text { background }\end{array}$ & $\begin{array}{c}S A A \\
\text { nodule }>B G\end{array}$ & GS overexpression & $\begin{array}{l}\text { Glypican-3 } \\
\text { expression }\end{array}$ \\
\hline $\begin{array}{l}\text { SAA-positive neoplasms } \\
\text { in alcoholic cirrhosis }\end{array}$ & 7 & $\begin{array}{c}7(100 \%)^{\mathrm{a}-\mathrm{c}} \\
6 / 1 / 0 / 0\end{array}$ & $1(14 \%)$ & $7(100 \%)^{a-d}$ & $0^{\mathrm{c}, \mathrm{d}}$ & $0^{\mathrm{c}}$ \\
\hline Focal nodular hyperplasia & 5 & $\begin{array}{l}1(20 \%) \\
0 / 0 / 1 / 4\end{array}$ & $1(20 \%)$ & 0 & $0^{\mathrm{c}, \mathrm{d}}$ & $0^{\mathrm{c}}$ \\
\hline Dysplastic nodule & 18 & $\begin{array}{c}10(56 \%) \\
0 / 2 / 8 / 8\end{array}$ & $4(22 \%)$ & $1(6 \%)$ & $3(17 \%)^{\mathrm{c}, \mathrm{d}}$ & $0^{\mathrm{c}}$ \\
\hline HCC, alcoholic & 18 & $\begin{array}{c}12(67 \%) \\
2 / 4 / 6 / 6\end{array}$ & $7(39 \%)$ & $6(33 \%)$ & $14(78 \%)$ & $16(89 \%)$ \\
\hline HCC, NAFLD & 7 & $\begin{array}{l}4(57 \%) \\
0 / 2 / 2 / 3\end{array}$ & $2(29 \%)$ & $2(29 \%)$ & $6(86 \%)$ & $5(71 \%)$ \\
\hline HCC, viral & 29 & $\begin{array}{l}11(38 \%) \\
0 / 2 / 9 / 18\end{array}$ & $18(62 \%)$ & 0 & $18(62 \%)$ & $23(79 \%)$ \\
\hline
\end{tabular}

Abbreviations: BG, background liver; F, female; GS, glutamine synthetase; HCC, hepatocellular carcinoma; M, male; NAFLD, non-alcoholic fatty liver disease; SAA, serum amyloid A.

${ }^{\mathrm{a}} P<0.01$ vs focal nodular hyperplasia.

${ }^{\mathrm{b}} P<0.01$ vs dysplastic nodule.

${ }^{\mathrm{c}} P<0.01$ vs HCC, alcoholic and viral.

${ }^{\mathrm{d}} P<0.05$ vs HCC, NAFLD.

In all, 15 of 17 nodules histologically examined were serum amyloid A-positive hepatocellular neoplasms (Figure 1). Hepatocellular neoplasms in patients 6 and 7 had been reported as benign hypervascular hepatocellular nodules by our institute. ${ }^{11}$ These serum amyloid A-positive hepatocellular neoplasms 

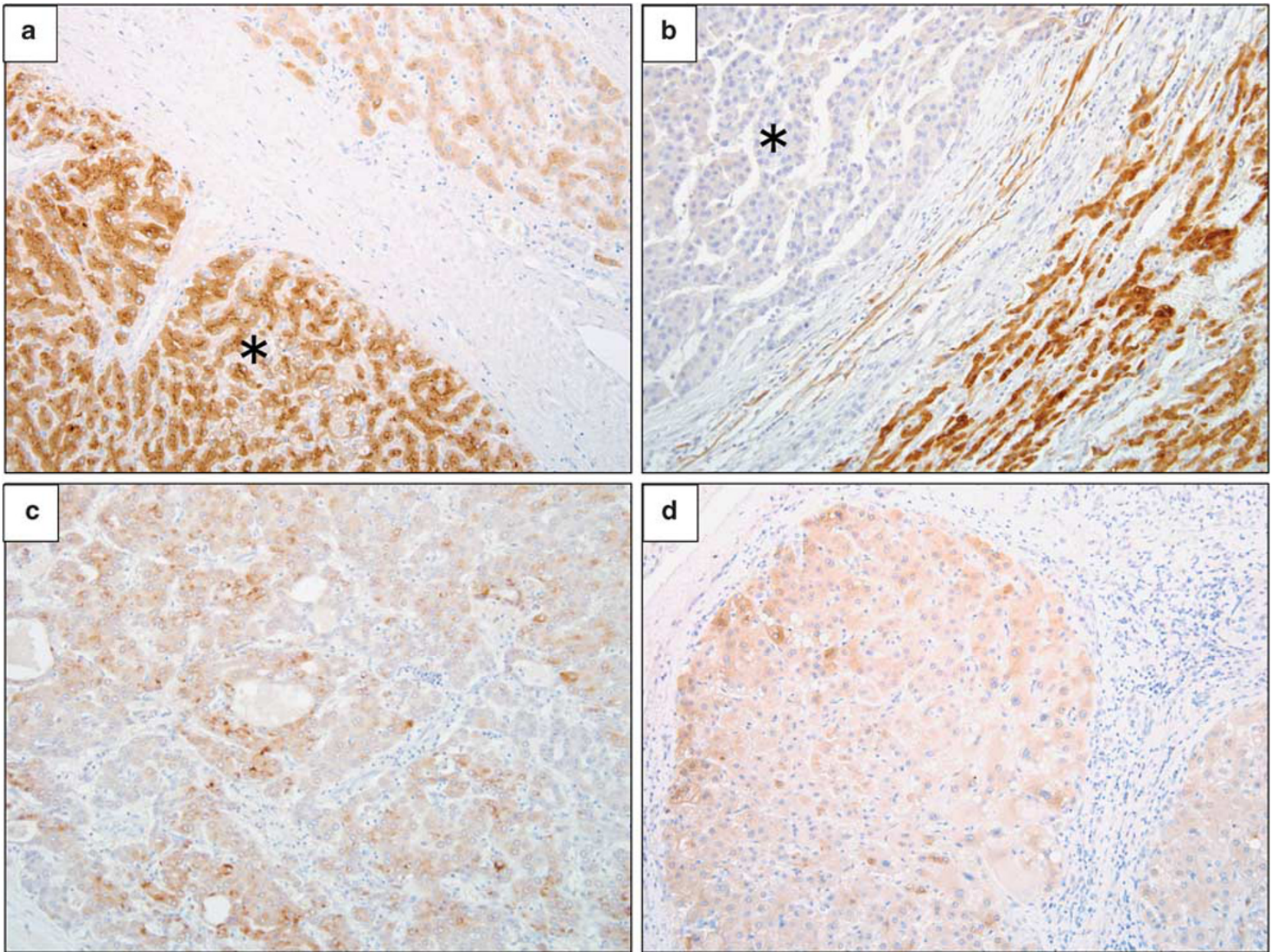

Figure 2 Various immunoreactivities for serum amyloid A in control hepatocellular nodules and the background livers. (a) Hepatocellular carcinoma in alcoholic cirrhosis. The carcinoma cells show a strong immunoreactivity for serum amyloid A $\left({ }^{*}\right)$, whereas the background liver shows weak immunoreactivity. Immunostaining for serum amyloid A, $\times 200$. (b) Hepatocellular carcinoma in chronic hepatitis B and cirrhosis. The carcinoma cells show no immunoreactivity for serum amyloid A $\left(^{*}\right)$, whereas the background liver shows immunoreactivity for serum amyloid A. Immunostaining for serum amyloid A, $\times 100$. (c) Hepatocellular carcinoma in chronic hepatitis $\mathrm{C}$ and cirrhosis. The carcinoma cells show focal and rather weak immunoreactivity for serum amyloid A. Immunostaining for serum amyloid A, $\times 200$. (d) The background liver of hepatocellular carcinoma shown in c. Hepatocytes in the background liver show focal and weak immunoreactivity for serum amyloid A, similarly to hepatocellular carcinoma. Immunostaining for serum amyloid A, $\times 200$.

showed increased cellular density, inflammatory infiltrate, sinusoidal dilatation, a ductular reaction, and thick arteries to various degrees (Figure 1). These serum amyloid A-positive hepatocellular neoplasms showed rather mild cellular atypia and no reduction of reticulin fibers. These histological and immunohistochemical features in serum amyloid A-positive hepatocellular neoplasms were the same as those in inflammatory hepatocellular adenoma. The remaining two nodules were diagnosed as benign hyperplastic hepatocellular nodules resembling focal nodular hyperplasia showing map-like immunostaining for glutamine synthetase and negative for serum amyloid $\mathrm{A}$. The association of hepatocellular carcinoma was not seen in any case examined. All background liver showed established micronodular cirrhosis, consistent with alcoholic cirrhosis (Figure 1). Mild steatosis was found in the background liver in two patients.

\section{Immunoreactivity for Serum Amyloid A, Glutamine Synthetase and Glypican-3 in Serum Amyloid A-Positive Hepatocellular Neoplasms and Control Benign and Malignant Hepatocellular Nodules}

Table 4 summarizes the immunoreactivity for serum amyloid A, glutamine synthetase, and glypican-3 in the groups examined. As described above, the immunoreactivity for serum amyloid A in serum amyloid A-positive hepatocellular neoplasms was extensively and significantly higher, than other control hepatocellular nodules $(P<0.01)$. It should be noted that two hepatocellular carcinomas in alcoholic liver diseases showed extensive immunoreactivity for serum amyloid A, similarly to serum amyloid A-positive hepatocellular neoplasms (Figure 2). Focal immunoreactivity for serum amyloid A was observed in about a half of the control groups (Figure 2). Background livers also showed focal 

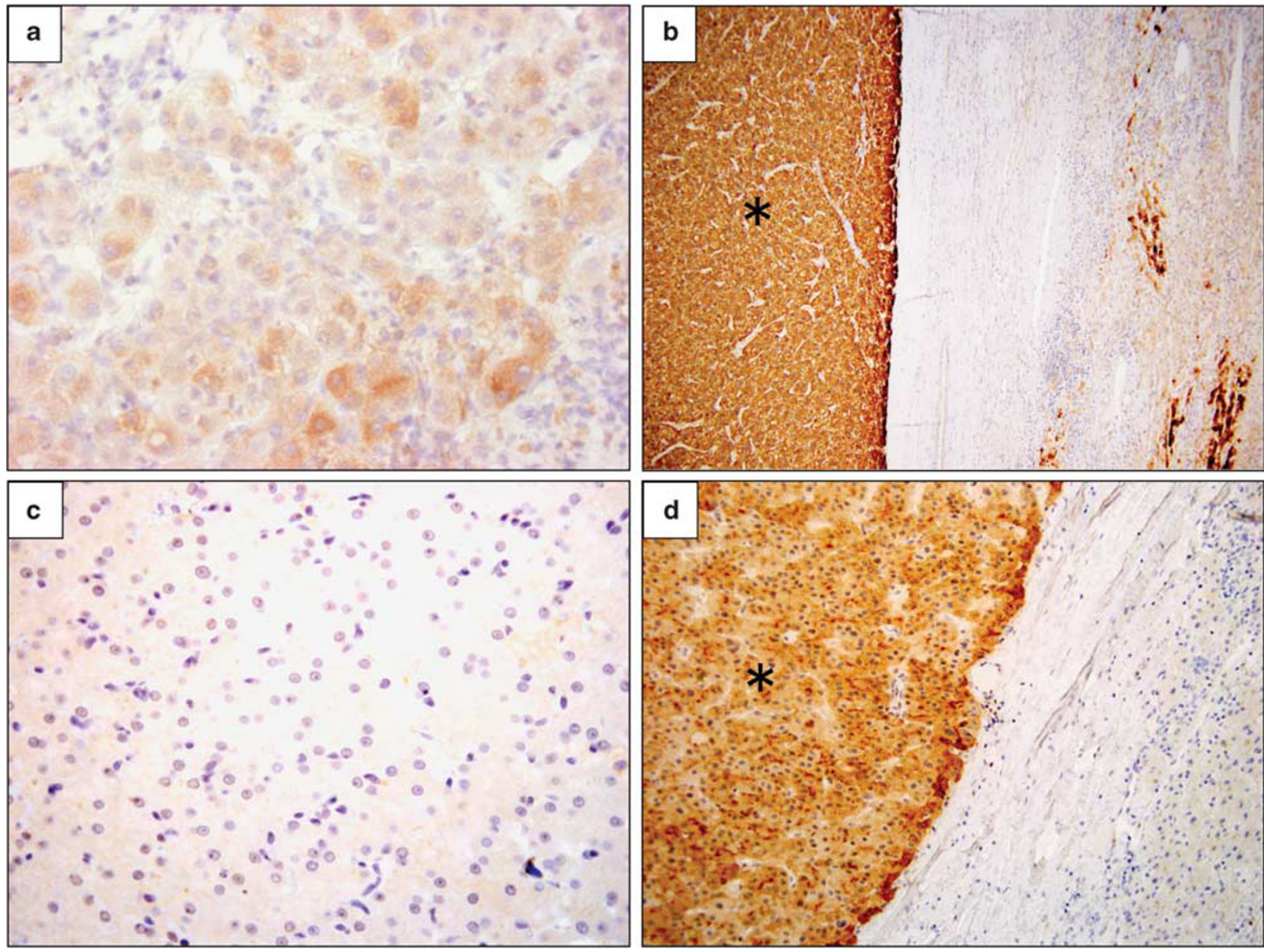

Figure 3 Immunoreactivity for glutamine synthetase and glypican-3 in serum amyloid A-positive hepatocellular neoplasms in alcoholic cirrhosis and control hepatocellular nodules. (a) The cells in the serum amyloid A-positive nodule in alcoholic cirrhosis show weak immunoreactivity for glutamine synthesis. Immunostaining for glutamine synthetase, $\times 400$, patient 4 . (b) Hepatocellular carcinoma shows strong and diffuse immunoreactivity for glutamine synthetase (*), regarded as glutamine synthetase overexpression. The background liver shows focal immunoreactivity for glutamine synthetase. Immunostaining for glutamine synthetase, $\times 100$. Hepatocellular carcinoma in chronic hepatitis C. (c) The cells in serum amyloid A-positive nodule in alcoholic cirrhosis show no immunoreactivity for glypican-3. Immunostaining for glypican-3, $\times 400$, patient 4 . (d) Hepatocellular carcinoma shows extensive immunoreactivity for glypican-3 $(*)$. In contrast, the background liver shows no reactivity for glypican-3. Immunostaining for glypican-3, $\times 200$. Hepatocellular carcinoma in chronic hepatitis B.

immunoreactivity for serum amyloid A in all groups to various degrees (Figure 2). There was no difference between hepatocellular nodules and background livers in the immunoreactivity for serum amyloid A in most control groups. All serum amyloid A-positive hepatocellular neoplasms, a dysplastic nodule, six hepatocellular carcinomas in alcoholic liver diseases, and two hepatocellular carcinomas in non-alcoholic fatty liver disease showed markedly more staining for SAA than the background liver (Table 4).

The serum amyloid A-positive hepatocellular neoplasms did not show overexpression of glutamine synthetase or immunoreactivity for glypican-3 at all (Figure 3; Table 4). In contrast, most hepatocellular carcinomas showed overexpression of glutamine synthetase and immunoreactivity for glypican-3, irrespective of background diseases (Figure 3; Table 4). Focal nodular hyperplasias showed a map-like immunoreactivity for glutamine synthetase as shown previously, ${ }^{8}$ and no focal nodular hyperplasia showed overexpression of glutamine synthetase. Focal nodular hyperplasias showed no immnoreactivity for glypican-3. In all, 3 of 18 dysplastic nodules showed overexpression of glutamine synthetase, whereas no dysplastic nodules showed immunoreactivity for glypican-3 (Table 4). Glutamine synthetase overexpression was significantly more frequent in hepatocellular carcinoma in all groups than in serum amyloid A-positive hepatocellular neoplasms, focal nodular hyperplasia, and dysplastic nodules (Table 4). The expression of glypican-3 was significantly more frequent in hepatocellular carcinoma in patients with alcoholic liver disease and viral hepatitis than in serum amyloid A-positive hepatocellular neoplasms, focal nodular hyperplasias, and dysplastic nodules (Table 4). 


\section{Discussion}

This study clearly demonstrated a distinct group of hepatocellular nodules showing strong immunoreactivity for serum amyloid A and histological features of inflammatory hepatocellular adenoma in patients with alcoholic cirrhosis. All of the serum amyloid A-positive hepatocellular neoplasms were detected as hypervascular hepatocellular nodules in the imaging study and some had been histologically diagnosed as hyperplastic hepatocellular nodules in a previous examination. These serum amyloid Apositive hepatocellular neoplasms showed different immunoreactivity for serum amyloid A, glutamine synthetase, and glypican-3 from focal nodular hyperplasia, dysplastic nodule, and hepatocellular carcinoma arising in alcoholic, non-alcoholic fatty liver disease, and viral hepatitis, and no evidence of malignancy. Taken together, it is conceivable that serum amyloid A-positive hepatocellular neoplasms may be a special type of inflammatory hepatocellular adenomas arising in alcoholic cirrhosis, as we have reported. ${ }^{8}$ To confirm proof of monoclonality or demonstration of gain of function mutations for gp130 will be mandatory in further studies. As these serum amyloid A-positive hepatocellular neoplasms showed several histological features resembling inflammatory hepatocellular adenomas, this lesion was not regarded as either a mere regenerative nodule or a mere dysplastic nodule.

There has been no other study describing inflammatory hepatocellular adenomas arising in alcoholic cirrhosis, to our knowledge. It is well known that hepatocellular carcinoma arises in various advanced liver diseases, including alcoholic diseases, whereas hepatocellular adenoma generally arises in the absence of significant fibrosis and background liver disease. ${ }^{2}$ A liver lesion with a solid mass arising in a fibrotic/cirrhotic background is not generally thought to be a hepatocellular adenoma, as shown in the diagnostic algorithm proposed in the WHO classification 2010. ${ }^{2}$ However, this study suggests the possibility of inflammatory hepatocellular adenoma arising in advanced alcoholic disease, in conflict with the diagnostic algorithm. ${ }^{2}$ In this sense, serum amyloid A-positive hepatocellular neoplasms, possibly inflammatory hepatocellular adenoma, may be a novel type of hepatocellular adenoma. The associated factors include alcohol use, tobacco use, elevated BMI (obesity), and fatty liver disease in inflammatory hepatocellular adenomas. $^{2,3,12}$ Reportedly, the patients were more frequently exposed to alcohol in inflammatory hepatocellular adenoma, than in hepatocyte nuclear factor $1 \alpha$-inactivated hepatocellular adenomas, ${ }^{3}$ therefore, it may not be surprising that both inflammatory hepatocellular adenoma and cirrhosis develop at the same time in alcoholic patients.

Distinct immunoreactivity for serum amyloid A is a critical characteristic defining serum amyloid A-positive hepatocellular neoplasms in this study as well as inflammatory hepatocellular adenomas. ${ }^{2,3,12}$ It is well known that IL- $1 \beta$ and IL-6 induce the expression of serum amyloid $\mathrm{A}$ in hepatocytes and hepatocellular carcinoma cell lines; $;^{13}$ however, there is no study addressing the expression of serum amyloid $\mathrm{A}$ in hepatocellular carcinoma and benign hepatocellular nodules such as focal nodular hyperplasia, to our knowledge. This study disclosed for the first time that immunoreactivity for serum amyloid A was seen in benign and malignant hepatocellular nodules to various degrees. Hepatocytes in the background livers, especially in viral hepatitis, also showed focal immunoreactivity for serum amyloid A. It is likely that inflammatory or other conditions induce the expression of serum amyloid A in hepatocellular nodules and the background livers; therefore, positive immunoreactivity for serum amyloid A itself may not be a specific marker for serum amyloid A-positive hepatocellular neoplasms. However, immunoreactivity for serum amyloid A was significantly more extensive in serum amyloid A-positive hepatocellular neoplasms than in other control hepatocellular nodules. Furthermore, it should be noted that all serum amyloid Apositive hepatocellular neoplasms showed markedly more immunoreactivity for serum amyloid A than background livers. It is plausible that the markedly higher expression of serum amyloid A occurred because of genetic changes in serum amyloid Apositive hepatocellular neoplasms. A similar pattern was exceptionally observed in one dysplastic nodule, six hepatocellular carcinomas in alcoholic liver diseases, and two hepatocellular carcinomas in non-alcoholic fatty liver disease. Taken together, the markedly greater immunoreactivity for serum amyloid A compared with the background liver may be critical for the diagnosis of serum amyloid A-positive hepatocellular neoplasms, probably inflammatory hepatocellular adenomas.

Sometimes, it is difficult to make a differential diagnosis between hepatocellular carcinoma and benign hepatocellular lesions such as hepatocellular adenoma. In addition to histological findings, a reduction of reticulin fiber, glutamine synthetase overexpression, and the expression of glypican-3 are used as markers of hepatocellular carcinoma. None of the serum amyloid A-positive hepatocellular neoplasms was diagnosed as hepatocellular carcinoma according to the pattern of these markers in this study; however, the possibility cannot be excluded that these serum amyloid A-positive hepatocellular neoplasms might be extremely welldifferentiated hepatocellular carcinomas.

Interestingly, some hepatocellular carcinomas arising in alcoholic cirrhosis and non-alcoholic fatty liver disease showed distinct immunoreactivity for serum amyloid A, similarly to serum amyloid Apositive hepatocellular neoplasms. The associated factors in inflammatory hepatocellular adenomas included alcohol use, tobacco use, elevated BMI (obesity), and fatty liver disease; ${ }^{2,3,12}$ therefore, this 
type of hepatocellular carcinoma may develop through a common pathway with serum amyloid A-positive hepatocellular neoplasms, probably inflammatory hepatocellular adenomas. In other words, serum amyloid A-positive hepatocellular neoplasms/inflammatory hepatocellular adenomas arising in alcoholic patients may be precursor lesions of hepatocellular carcinoma. Interestingly, Farges et $a l^{14}$ have recently reported changing trends in the malignant transformation of hepatocellular adenoma. The prevalence of malignancy in hepatocellular adenoma was 10 times more frequent in men than in women in their study, ${ }^{14}$ and they propose that management of hepatocellular adenoma should primarily be based on gender. Although oral contraception is a classical cause of hepatocellular adenoma in women but a marginal cause of hepatocellular carcinoma, metabolic syndrome appears to be an emerging condition associated with malignant transformation of hepatocellular adenoma in men, and is the likely predisposing condition for hepatocellular carcinoma in this setting. ${ }^{14}$ In fact, hepatocellular adenomas in men were distributed almost exclusively in the inflammatory hepatocellular adenoma subgroup in previous studies. ${ }^{3}$ Although no serum amyloid A-positive hepatocellular neoplasms were associated with hepatocellular carcinoma in this study, it is conceivable that serum amyloid A-positive hepatocellular neoplasms/inflammatory hepatocellular adenomas arising in men with alcoholic cirrhosis may have a higher risk of malignant transformation.

Several studies have demonstrated a hypervascular liver nodule showing similar imaging findings to hepatocellular carcinoma; hypervascular hepatocellular nodules, so-called focal nodular hyperplasialike nodules, occur in severe alcoholic fibrosis or cirrhosis. ${ }^{11,15,16}$ This study disclosed that some such focal nodular hyperplasia-like nodules may be serum amyloid A-positive hepatocellular neoplasms/inflammatory hepatocellular adenomas. In fact, two of seven patients (patients 6 and 7) with serum amyloid A-positive hepatocellular neoplasms in this study had been diagnosed previously as hypervascular hyperplastic nodule/focal nodular hyperplasia-like nodules in our institute. ${ }^{11}$ Focal nodular hyperplasia-like nodules associated with alcoholic fibrosis or cirrhosis in this study and our previous study ${ }^{8}$ showed a typical map-like staining pattern of glutamine synthetase. ${ }^{2,17}$ Differentiation of hepatocellular adenoma and focal nodular hyperplasia may be important because focal nodular hyperplasia generally does not require treatment and follow-up, whereas hepatocellular adenoma does. Further studies including imaging studies are mandatory to characterize serum amyloid A-positive hepatocellular neoplasms for assessment of the outcome of the nodules.

In conclusion, we have presented seven patients with serum amyloid A-positive hepatocellular neoplasms, probably inflammatory hepatocellular adenomas arising in alcoholic cirrhosis. This study highlights a characteristic group of hepatocellular nodules arising in alcoholic cirrhosis, which share features with inflammatory hepatocellular adenomas. These serum amyloid A-positive hepatocellular neoplasms may be a new type of inflammatory hepatocellular tumors having potential for malignant transformation in alcoholic patients.

\section{Acknowledgement}

This study was supported in part by a Grant-in-Aid for Scientific Research (C) from the Ministry of Education, Culture, Sports and Science and Technology of Japan (21590366).

\section{Disclosure/conflict of interest}

The authors declare no conflict of interest.

\section{References}

1 Rooks JB, Ory HW, Ishak KG, et al. Epidemiology of hepatocellular adenoma. The role of oral contraceptive use. JAMA 1979;242:644-648.

2 Bioulac-Sage P, Balabaud C, Wanless I. Focal nodular hyperplasia and hepatocellular adenoma. In: Bosman F, Carneiro F, Hruban H, Theise $\mathrm{N}$ (eds) WHO Classification of Tumours of the Digestive System 4th edn, Vol., IARC: Lyon 2010, pp 198-204.

3 Bioulac-Sage $\mathrm{P}$, Laumonier $\mathrm{H}$, Couchy $\mathrm{G}$, et al. Hepatocellular adenoma management and phenotypic classification: the Bordeaux experience. Hepatology 2009;50:481-489.

4 Zucman-Rossi J, Jeannot E, Nhieu JT, et al. Genotypephenotype correlation in hepatocellular adenoma: new classification and relationship with HCC. Hepatology 2006;43:515-524.

5 Bioulac-Sage $\mathrm{P}$, Rebouissou S, Thomas C, et al. Hepatocellular adenoma subtype classification using molecular markers and immunohistochemistry. Hepatology 2007;46:740-748.

6 Rebouissou S, Bioulac-Sage P, Zucman-Rossi J. Molecular pathogenesis of focal nodular hyperplasia and hepatocellular adenoma. J Hepatol 2008;48:163-170.

7 Konishi M, Ryu M, Kinoshita T, et al. A case report of liver cell adenoma. Acta Hepatol Jpn 1995;36:223-229.

8 Sasaki M, Yoneda N, Kitamura S, et al. Characterization of hepatocellular adenoma based on the phenotypic classification: the Kanazawa experience. Hepatol Res 2011;41:982-988.

9 Di Tommaso L, Destro A, Seok JY, et al. The application of markers (HSP70 GPC3 and GS) in liver biopsies is useful for detection of hepatocellular carcinoma. J Hepatol 2009;50:746-754.

10 Sasaki M, Ikeda H, Sato Y, et al. Decreased expression of Bmi1 is closely associated with cellular senescence in small bile ducts in primary biliary cirrhosis. Am J Pathol 2006;169:831-845.

11 Kobayashi S, Matsui O, Kamura T, et al. Imaging of benign hypervascular hepatocellular nodules in alcoholic liver cirrhosis: differentiation from hypervascular 
hepatocellular carcinoma. J Comput Assist Tomogr 2007;31:557-563.

12 Paradis V, Champault A, Ronot M, et al. Telangiectatic adenoma: an entity associated with increased body mass index and inflammation. Hepatology 2007;46: 140-146.

13 Ganapathi MK, May LT, Schultz D, et al. Role of interleukin-6 in regulating synthesis of C-reactive protein and serum amyloid A in human hepatoma cell lines. Biochem Biophys Res Commun 1988;157:271-277.

14 Farges O, Ferreira N, Dokmak S, et al. Changing trends in malignant transformation of hepatocellular adenoma. Gut 2011;60:85-89.
15 Terada T, Kitani S, Ueda K, et al. Adenomatous hyperplasia of the liver resembling focal nodular hyperplasia in patients with chronic liver disease. Virchows Arch A Pathol Anat Histopathol 1993;422: 247-252.

16 Nakashima O, Kurogi M, Yamaguchi R, et al. Unique hypervascular nodules in alcoholic liver cirrhosis: identical to focal nodular hyperplasia-like nodules? J Hepatol 2004;41:992-998.

17 Bioulac-Sage P, Laumonier H, Rullier A, et al. Overexpression of glutamine synthetase in focal nodular hyperplasia: a novel easy diagnostic tool in surgical pathology. Liver Int 2009;29:459-465. 